\title{
Unterstellte Leseschaften
}

\section{Dirk Knipphals Gelesen werden.}

Illusionen, Erfahrungen und Vermutungen eines Literaturredakteurs 


\section{Der Lesekreis}

Ein Essay über das Glück der gleichen Augenhöhe zwischen Autor*innen und Leser*innen (und die Probleme, die ihr entgegenstehen). Auch eine Hommage an den versierten Leser Michael Rutschky

Ich war einmal im Raum, als der Essayist Michael Rutschky, sich unbeobachtet wähnend, Zeitung las. -

Die Frage, für wen man Zeitung produziert, also welche Leserschaft man unterstellt, beantwortet man als Redakteur im tagtäglichen Redaktionsgeschäft ganz praktisch und ohne sich darüber groß Gedanken zu machen; man zehrt da, vielleicht lässt es sich so fassen, von seinen Intuitionen. Welches Thema zieht man hoch, welches lässt man aus? Welches Buch könnte interessant sein, welches eher fad? Welchen Text bebildert man, welchen nicht? Welche/n Rezensent*in mit welchem Profil fragt man an, wem sagt man ab? Jede einzelne dieser vielen alltäglichen Redaktionsentscheidungen konstruiert implizite Leser*innen. Das ist redaktioneller Alltag.

Insofern wundert es mich fast, in wie große Verlegenheit mich die Frage „Für wen machen Sie eigentlich Zeitung?" bringt, wenn sie, wie das gelegentlich geschieht, explizit an mich gestellt wird. Denn das tut sie. Sie bringt mich in Verlegenheit. Die Frage zielt schließlich nicht nur darauf, wer die Zeitung, an der ich mitwirke, tatsächlich kauft und idealerweise dann auch liest. Von der Leserschaft der taz habe ich auch eine ungefähre Vorstellung; da gibt es regelmäßige Leserbefragungen, deren Ergebnisse der Redaktion auch mitgeteilt werden. Die Frage zielt auf mehr. Es ist auch eine Frage nach dem professionellen Selbstverständnis und danach, welchen Sinn man seiner Tätigkeit gibt oder zumindest geben möchte. Was und wen willst du mit deiner Arbeit unterstützen? An welcher Gruppe willst du teilhaben? Auch solche Fragen schwingen in der Frage, für wen man Zeitung macht, mit.

Wenn sie mir gestellt wird, winde ich mich oft etwas. Und dann sage ich manchmal so etwas wie: „Ich mache Zeitung für Menschen, die gern lesen.“ Damit meine ich: für Menschen, die gern Zeitung lesen und die gern Romane lesen; schließlich bin ich Literaturredakteur. Das ist im Grunde eine unbefriedigende Antwort, ich weiß, denn 
gleich stellt sich die nächste Frage: Was heißt das? Aber man kann mit ihr ins Gespräch kommen. Und das Gespräch könnte zum Beispiel die Freude behandeln, die man empfindet, wenn es einem als Literaturredakteur gelingt, kleine Siege über die Marketingmaschine des Literaturbetriebs zu erringen, also, wenn man zum Beispiel daran beteiligt ist, Bücher in den Vordergrund zu rücken, die es verdienen, aber aus irgendwelchen Gründen nicht zu den Spitzentiteln der Saison zählen. Oder wenn es um Bücher, die durchgejubelt werden sollen, dann doch Debatten gibt, die zwar vielleicht nicht den Verkaufserfolg verhindern, wohl aber, dass das Buch auch noch als positiver Maßstab für den Stand von Gegenwartsliteratur gehandelt wird, wie es etwa bei Takis Würgers allzu schmissig geschriebenem Roman „Stella“ über ein reales jüdisches Frauenschicksal im Zweiten Weltkrieg der Fall zu werden drohte.

Das Gespräch könnte sich auch um den Wunsch drehen, aus der Distinktionsspielen rund um Literatur herauszutreten und um die Suche nach Verbündeten für dieses Projekt. Als Feuilletonredakteur ist man, diffus im sozialen Umfeld, aber auch ganz konkret in Leserzuschriften und Redaktionskonferenzen mit offenbar langlebigen Vorurteilen konfrontiert, die sich, wie ich denke, auch im Umgang mit Literatur spiegeln. Im Wesentlichen umkreisen diese Vorurteile - ob positiv oder negativ konnotiert - die Ansicht, dass man sich auf den Feldern der Kultur für etwas Besseres halten kann als auf den Feldern reiner Unterhaltung, des Alltags oder auch des Gelderwerbs. Das tut, meine ich, ganz einfach auch dem Lesen nicht gut, weil es vom einzelnen Buch ablenkt. Aber es ist auf der anderen Seite wiederum auch sehr die Frage, ob man aus solchen Distinktionsspielen überhaupt heraustreten kann, und, noch ein bisschen perfider, es nicht sogar wiederum ein Privileg darstellt, wenn man daraus heraustreten kann. Die Distinktionsspiele rund um Bildungserwerb in der Aufstiegsgesellschaft nicht mitmachen zu müssen - das muss man sich auch leisten können.

Im Zuge des Gesprächs könnte ich dann auch von dem Moment erzählen, in dem ich Herrn Rutschky beim Zeitunglesen beobachtete; ein Augenblick, in dem sich für mich viele Erfahrungen rund um das Lesen bündelten. Ich möchte hier die Gelegenheit nutzen, das, was ich zu diesem Teil eines solchen Gesprächs beizutragen habe, einmal aufzuschreiben. Der 2018 verstorbene Essayist Michael Rutschky wird mich durch meinen Text also begleiten, aber ich werde ihn in seiner Person und seinem Werk nicht weiter vorstellen und setze dabei hoffentlich nicht zu viel voraus. Ich bin 
mir unsicher, wem der Name noch etwas sagt. Für Menschen, die sich über ihn informieren möchten, empfehle ich als Einstiege die Rezension des ersten Tagebuchbandes von Michael Rutschky, die der Historiker Philipp Felsch 2015 in der taz geschrieben hat ${ }^{1}$, und die Einführung über den sogenannten Rutschky-Kreis, die Stephan Wackwitz 2019 in der Zeit publizierte ${ }^{2}$. Da finden sich auch die ambivalenten Züge seiner Figur, die ich hier eher ausblende.

\section{ZEITUNGLESEN UND HACKORDNUNGSWELT}

Michael Rutschky und seine Frau Katharina Rutschky hatten fünf Tageszeitungen abonniert. Süddeutsche, Frankfurter Rundschau, Tagesspiegel, taz und Berliner Zeitung. Nicht die FAZ, mit Frank Schirmmacher war mal irgend etwas vorgefallen, was bad feelings hinterlassen hatte (Herr und Frau Rutschky konnten nachtragend sein). Falls es wichtige Texte in der FAZ gab, wurde er von seinem Freund Kurt Scheel, Ko-Herausgeber der Monatszeitschrift Merkur, zuverlässig darauf aufmerksam gemacht und mit einer Kopie versorgt. Die anderen Zeitungen aber wurden täglich von ihm selbst sorgfältig durchgearbeitet.

Und zwar hatte Herr Rutschky an diesem Tag die Zeitung - es war damals die FR, als sie noch das große Format hatte - als Doppelseite ausgebreitet vor sich auf dem Tisch liegen (bzw. auf der besonders breiten Fensterbank, wie es sie in den sogenannten Berliner Zimmern manchmal gibt). Bevor er einzelne Artikel las, verschaffte sich Herr Rutschky mit unruhigem Blick und ruckenden Kopfbewegungen Orientierung über die Doppelseite. Anordnung der Artikel. Blick auf Titelei: Thema des Artikels, Blick ans Ende und genauso wichtig: Autor, Autorin des Artikels. Und das für jeden einzelnen Text. Erst wenn diese Informationen eingeholt waren, ging es an das Lesen der einzelnen Stücke.

Was sucht er da?, habe ich mich gefragt -

\footnotetext{
1 Philipp Felsch: „Ich nehme den Wildtopf Diana. Zu den Tagebüchern des Essayisten Michael Rutschky“. In: taz - die tageszeitung, 1. 9. 2015.

2 Stephan Wackwitz: „Der Rutschky-Kreis“. In: Die Zeit. Nr. 33/19, 7. 8. 2019.
} 
Michael Rutschky war ein leidenschaftlicher Hundebesitzer. (Kinder sind ein ganz guter Hundeersatz, versicherte Frau Rutschky mir einmal wohlwollend; ein schönes Beispiel für die Freude an provozierenden Bonmots und pointierten Formulierungen, die in diesem Hause herrschte.) Und tatsächlich musste ich, als ich Herrn Rutschky Zeitung lesen sah, an einen Hund denken. So wie ein Hund sein Terrain aufmerksam und konzentriert mit der Nase sondiert, so schritt Michael Rutschky sein Terrain täglich lesend ab. Die Informationen, die er lesend sammelte, waren sein Lebensraum. Wer schreibt heute was? Welche Themen werden wie behandelt? Wo tauchen womöglich neue Themen auf? Wo tauchen neue Namen auf?

Möglicherweise hat Herr Rutschky selbst eine gewisse Ähnlichkeit seines Zeitungslesens mit dem Terrainsondieren seines Hundes gesehen. Als wir einmal spazieren waren und Pelo, sein damaliger Hund, an einem Laternenpfahl roch, hat Herr Rutschky gesagt: Sehen Sie, der Hund studiert gerade wieder seine Zeitung.

Ich glaube, die Szene hat mich auch deshalb so beeindruckt, weil sie ziemlich quer zu der Art und Weise liegt, wie in Zeitungsredaktionen über Leser*innen nachgedacht wird. Man muss sich gar nicht an die kommunikationstheoretisch naivsten Journalist*innen und Redakteur*innen (von denen es viele gibt) halten, um festzustellen, dass in Redaktionen Leser*innen sozusagen stillgestellt und als nur empfangende Rezipienten dargestellt werden. Auf Blattkritiken wird das eigene Leseverhalten oft umstandslos verallgemeinert. Und es wird davon ausgegangen, dass der Leser, die Leserin brav der hierarchisierenden Struktur folgt, die eine Zeitung vorgibt: also vorne anfängt zu lesen usw.

Anhand von Herrn Rutschky habe ich aber endgültig gelernt, wie aktiv der Vorgang des Lesens ist und dass Hierarchisierungen und Stillstellungen einen auf die falsche Fährte setzen. Salopp formuliert: Die Hunde, die das Terrain markieren, also die Autor*innen der Texte, sind bei ihm keine andere Art Hunde als die, die das dann rezipieren. Zwischen Autor*innen und Leser*innen herrscht also kein Autoritätsgefälle, es sind verschiedene Rollenübernahmen in einem gemeinsamen Spiel.

Ich glaube, dass Michael Rutschky mit seinem Selbstbild und seiner Praxis eines souveränen Lesers in Deutschland lange Zeit aus der gängigen Sicht auf Leser*innen 
herausgefallen ist und immer noch teilweise herausfällt. Ich glaube, in der Breite und wenn man sich aus dem literaturwissenschaftlichen Feld herausbewegt - wird nämlich sehr stark von einem Autoritätsgefälle zwischen Autor*innen und Leser*innen ausgegangen, und zwar bis heute.

Und dieses Autoritätsgefälle wird auch bewirtschaftet. Ratgeberbücher von Professoren, die einem sagen können, wie man Bildung erwerben kann, verkaufen sich gut.

Mir fällt in diesem Zusammenhang schnell eine Szene aus den ersten Kapiteln des Romans Stoner von John Williams ein, der 1965 geschrieben worden ist, gerade vor einigen Jahren aber erst in Deutschland noch einmal ein großer Überraschungserfolg war. Die Hauptfigur William Stoner, ein Farmerssohn, so geht die Szene, kommt auf dem College mit der Literatur in Berührung. Bei dem Englischprofessor Archer Sloane nehmen sie ein Sonett von Shakespeare durch. Archer Sloane fragt: „Über drei Jahrhunderte hinweg redet Mr Shakespeare mit Ihnen, Mr Stoner. Können Sie ihn hören?“ (Als Leser hört man an dieser Stelle etwas Donnerndes in dieser Stimme.)

Stoner kann darauf nicht gleich antworten. Aber etwas geschieht mit ihm. Kurz darauf sieht er sich auf dem Campus unter seinen Kommilitonen um. Und dann heißt es: „Er wurde sich in einem zuvor ungekannten Maße seiner selbst bewusst.“ Ein Erweckungserlebnis durch Literatur. Der klassische Autor also als die Autorität, die über die Jahrhunderte hindurch den Leser, die Leserin zu erwecken vermag.

Ich glaube, der Erfolg der Neuübersetzung dieses Romans hängt vor allem auch mit dieser Szene zusammen. Viele Leserinnen und Leser lassen sie sich immer noch gern erzählen, die Geschichte, dass man durch Literatur zu einem anderen Menschen werden kann. Der Buchhandel lebt davon. Statt des Namens Archer Sloane kann man als stellvertretende Autorität, die ihre Aura durch die Autorität der Literatur verliehen bekommt, viele Namen einsetzen.

Wer etwa dabei war, als Marcel Reich-Ranicki den großen Erfolg seiner Autobiografie Mein Leben auf der Frankfurter Buchmesse zelebrierte, wird dies Bild nicht wieder vergessen. Er war damals, ich meine es war 1999, im Palmengarten, einer alten Villa mit hochherrschaftlicher Treppe, und oben auf der Treppe stand 
Reich-Ranicki und freute sich darüber, mehr Bücher verkauft zu haben als Martin Walser in dieser Saison.

Oder, ein anderes Bild, Michael Krüger, der ehemalige Verleger des Hanser-Verlages, der auf einem Festvortrag fünfzehn Jahre später, auch auf der Frankfurter Buchmesse, eigentlich nur den einen Gedanken, das aber mit tief tönender Stimme intonierte, dass, wenn er erst einmal kein Verleger mehr sein wird, eigentlich die ganze Literatur den Bach heruntergeht -

Was ich sagen möchte: Die deutsche Literaturlandschaft war lange Zeit und eigentlich noch bis vor kurzem eine autoritäre Hackordnungswelt, und in diese Konstellation eingebunden war die Leser*in. Das galt nicht nur für konservative Leser*innen, sondern auch für die Gegenkultur. Auch die anti-autoritären Leser*innen glaubten an die Autorität der Bücher, Stichwort Adorno.

Während ich das aufschreibe, fällt mir auf, dass das jetzt vielleicht etwas ungerecht pointiert ist, aber an dem Wort Hackordnungswelt möchte ich unbedingt festhalten. Man schaue sich einmal die alten Buchmessenbeilagen der $F A Z$ an, anhand der Reihenfolge und Platzierung der einzelnen Besprechungen konnte man sehr genau die Hierarchie innerhalb der Redaktion ablesen. Literaturchef schreibt den Aufmacher, Stellvertreter auf der zweiten Seite, für altgediente Granden gab es Ehrenplätze mit wenigstens großen Bebilderungen usw. Das war fein säuberlich hierarchisch durchkomponiert. Und Verlagskataloge funktionierten - mit der Ausnahme des zu Lebzeiten von Siegfried Unseld alphabetisch aufgebauten Suhrkamp-Katalogs - genauso.

Ich glaube nun, dass es auch dieser Hintergrund war, vor dem das Zeitungslesen von Michael Rutschky so einen Eindruck auf mich machte. Seine Art, Zeitung zu lesen, markiert ziemlich genau das Gegenteil eines bildungsbürgerlichen Fetischcharakters, eines Erweckungserlebnisses oder einer autoritären Überhöhung der Autor*inneninstanz. Er sucht den Gebrauchswert. Und wir sehen bei ihm die Leser*innen mitten drin in ihrer aktiven hermeneutischen Arbeit.

Leitartikel oder auch Essays, die Leser*innen erwecken oder auch nur wachrütteln wollen - in der Art von: Wir müssen hier und jetzt handeln, sonst wird die Gesellschaft 
untergehen - hat Michael Rutschky, wie man in vielen seiner Essays nachlesen kann, nie recht ernst genommen, teilweise sogar verachtet. Was er im Lesen sucht, ist Material, Futter für die eigene hermeneutische Arbeit, die Realität zu verstehen. Dazu braucht er Anschauungsmaterial, aber auch Denkansätze, Thesen - Michael Rutschky nennt das in seinen eigenen Essays manchmal auch Schemata -, um dieses Anschauungsmaterial anzuordnen. Seine Art zu lesen ist in eine ständige Deutungsanstrengung, was in der Welt vor sich geht, verstrickt. Und diese Deutungsarbeit lässt er sich von niemandem nehmen.

Festhalten möchte ich an dieser Stelle, dass Michael Rutschky es nie nötig hatte, sich von vermeintlich weniger wertvollen Textformen oder Rezeptionsverhalten abzugrenzen, wie es viele Anhänger einer autoritären Sicht auf Literatur - die Archer Sloanes von heute - gelegentlich tun.

Über Fernsehserien konnte man sich mit Herrn Rutschky prima austauschen. Von den Sopranos etwa, längst ein Klassiker, habe ich zum ersten Mal von Michael Rutschky gehört, und zwar sehr früh, da musste man sich die DVDs noch aus den USA schicken lassen. Was das Internet betrifft, war er zwar ein very late adopter, und er fremdelte auch mit den Formen der im Netz üblichen Kommunikation; Mails schrieb er wie kurze Briefe oder auch wie Telegramme. Aber er hat zuletzt noch einen Blog betrieben, Das Schema hieß er. Überhaupt finde ich seine Art, Zeitung zu lesen, sich dabei nicht beeindrucken zu lassen, und die Ansätze und Argumente pointiert herauszuarbeiten, inzwischen manchmal auf Twitter wieder. Aber das ist eine andere Geschichte -

Jedenfalls war er in meinem Lebenslauf gerade dadurch wichtig, dass er aus der Hackordnungswelt des literarischen Felds ein Stück weit herausfiel.

\section{PRIVILEGIEN DER ZUGEHÖRIGKEIT}

Neben Stoner von John Williams kann einem zu dem sondierenden Zeitungslesen Michael Rutschkys noch eine zweite Romanszene zur Kontrastierung einfallen. Ich meine den Debütroman Streulicht von Deniz Ohde. Ziemlich genau in der Mitte dieses Romans schließt die Ich-Erzählerin ein Schülerabonnement der Wochenzeitung Die Zeit ab, als Werbegeschenk erhält sie eine Umhängetasche, damit läuft sie dann herum. 
Warum sie das tut, ist der Erzählerin selbst vollkommen klar. Sie möchte damit Zugehörigkeit demonstrieren zu bürgerlichen Sphären, sie möchte zeigen, dass sie nicht wirklich am Rande der Gesellschaft steht. Zuvor musste sie das Gymnasium verlassen. Von ihrem Elternhaus hat sie keine Unterstützung zu erwarten. Jetzt versucht sie es auf dem zweiten Bildungsweg auf einer Abendschule. Das demonstrative Zeit-Abonnement soll zeigen - auch ihr selbst zeigen -, dass sie dagegen eigentlich aufs Gymnasium gehört.

Das Interessante im Zusammenhang dieses Textes und gewissermaßen genau das Gegenteil der Szene mit Michael Rutschky: Die Zeitung liest die Erzählerin dabei gar nicht wirklich. Die Tasche und die Tatsache, dass die Zeitungs-Exemplare zu ihr nach Hause geliefert werden, reichen ihr aus. Die Zeit funktioniert wie ein Fetisch ihres Bildungswillens.

Ich habe diesen Roman sehr, sehr gern gelesen. Und über diese Episode mit der Umhängetasche der Zeit war ich geradezu dankbar. Ich glaube, dass sie einen real vorhandenen Rezeptionsmodus sehr gut abbildet.

Dass wir tatsächlich in einer Gesellschaft leben, die soziale Aufstiege ermöglicht, kann man mit Blick auf die real schlechten Aufstiegsbedingungen etwa für Arbeiterkinder zwar bestreiten, aber wir leben doch in einer Gesellschaft, in der die Elterngeneration oder die Großelterngeneration am Band gearbeitet hat oder unter Tage, während man selbst in irgendeiner Funktion hinter einem Computerbildschirm sitzt. Demonstrierter Bildungswille gehört in ihr zu den verbreiteten Taktiken, mit den komplizierten Anforderungen, die sich aus ihr ergeben, zurechtzukommen.

Die interessante Frage ist jetzt vielleicht, warum es funktioniert, dass die Erzählerin, ohne zu lesen, Zugehörigkeitswillen zu bürgerlichen Kreisen demonstrieren kann. Es funktioniert wiederum aufgrund eines starken Autoritätsgefälles. Bei Deniz Ohde fällt ausdrücklich das einschlägige Stichwort: Der Vater der Erzählerin redet „ehrfürchtig“ über Die Zeit. 
Ehrfurcht. Demut. Der Autor, die Autorin und die Zeitung, in der er/sie schreibt, als Repräsentant*in einer Autorität, deren Aura auf einen abstrahlt, wenn man nur ihre Texte mit sich herumträgt -

Wie lässt sich die Erzählerin bei Deniz Ohde nun mit Michael Rutschky und seiner Form des Lesens in Beziehung setzen?

Selbstverständlich bezeichnet die Form des Lesens bzw. eben Nichtlesens bei Deniz Ohde eine defizitäre Form des Umgangs mit Texten. Aber immerhin ist dieser Erzählerin, wenigstens im Nachhinein, im Abstand des Erzählens, sehr bewusst, was sie da macht. Sie möchte Zugehörigkeit demonstrieren. Sie möchte sich nicht auf ihre Herkunft festlegen lassen. Und sie verbrämt das nicht, wie es die Romandramaturgie bei Stoner vorsieht, zum Erweckungserlebnis. Sie ist ehrlich.

Wenn man genau hinsieht, erscheint die Art des souveränen Lesens, die Michael Rutschky demonstriert, von der Erzählerin bei Deniz Ohde aus auch als Privileg. Die gleiche Augenhöhe zwischen Leser und Autor, die er erreicht hat, ist ja etwas, was der Erzählerin bei Deniz Ohde nicht zuhanden ist. Ihre Demut und Schüchternheit - vom Gymnasium musste sie gehen, nicht weil sie eine wirklich schlechte Schülerin war, sondern weil sie nie etwas gesagt hat - ist ein Reflex dessen, dass ihr diese Augenhöhe als Arbeiterkind gar nicht zugestanden wird.

Und es gibt eine Gemeinsamkeit, die mich interessiert: eben der Wille zur Zugehörigkeit zur Sphäre des Lesens und Schreibens. Nur dass die Erzählerin bei Deniz Ohde ihn von einem Außen aus formuliert, während Michael Rutschky ihn im Inneren dieser Sphäre ausagiert. Während die Erzählerin bei Deniz Ohde sozusagen von außen an die Tür klopft, hat Michael Rutschky das alte Feuilleton gewissermaßen bewohnt.

\section{ENTAURATISIERUNG UND EMANZIPATION}

Dieses Feuilleton und überhaupt der Umgang mit Texten haben sich in den vergangenen Jahren stark verändert. Ich meine jetzt nicht die Schrumpfung von Umfängen oder der schieren Anzahl von Rezensionen, die immer mal wieder besorgt thematisiert werden. Ich meine die Veränderungen im Zugang zur Literatur. 
Am anschaulichsten kann ich mir das anhand der Leipziger Buchmesse deutlich machen. Die ersten Leipziger Buchmessen, auf denen ich gewesen bin, 1994, 1995, waren im Kern noch geschlossene und dabei auch hierarchische Veranstaltungen mit klaren Hackordnungen, was die Aufmerksamkeiten und die Sprecherpositionen betrifft. Durch Zufall war ich in demselben Hotel wie Lew Kopelew untergebracht; er hielt, so habe ich es empfunden, Hof wie ein Gegenfürst. Die Figur des Dissidenten war noch bis in die neunziger Jahre hinein mit mächtigen Kraftlinien aufgeladen.

In der Literatur herrschten die Platzhirsche: Grass, Walser, Enzensberger, Handke, in der Literaturkritik auch: klar, Reich-Ranicki. Selbstverständlich gab es auch Gegenhelden und Nischenfürsten, aber die symbolischen Kämpfe wurden doch um die Granden ausgetragen.

Was sich inzwischen aber durchgesetzt hat, ist, die Buchmesse mit Talkshow-Elementen zu durchsetzen. Interessant für viele Besucher*innen ist diese hohe Dichte an Debatten, Buchvorstellungen und Diskussionen, die man sich mittlerweile auf der Leipziger Messe abholen kann. Es sind immer mehr geworden. Ob Blaues Sofa oder Deutschlandfunk, Leipziger Volkszeitung, Börsenverein, die Messe selbst oder auch die taz: Überall werden Bücher vorgestellt, Autorinnen promotet, Themen gesetzt. Die Messe ist inzwischen so etwas wie eine Dauertalkshow.

Was immer man davon hält - viele dieser Debatten bleiben bei der Promo, andere sind aber auch immer wieder überraschend gut -, verbunden ist diese Entwicklung mit einer Zunahme möglicher Sprecherpositionen. Und damit auch mit einer Zunahme möglicher Zugänge zur Literatur. Das Ergebnis ist ambivalent. Auf der für mich negativen Seite: Der Autor wird als Star bei solchen Veranstaltungen teilweise vorgeführt. Aber auf der positiven Seite: Diese Vielfalt ermöglicht Emanzipationschancen.

Was ich Herrn Rutschky nun hoch anrechne, ist, dass er andere Formen des Willens zur Zugehörigkeit zum literarischen Feld eigentlich niemals abgewertet hat; während viele andere Vertreter des alten Literaturbetriebs in der Zunahme von Sprecherpositionen und Zugängen oft nur Verflachung und Verfall der Sitten gesehen haben. 
Ich frage mich nun, warum Michael Rutschky als einer der wenigen seiner Generation da keine Abgrenzungsprobleme hatte.

Der Punkt ist, glaube ich, dass Michael Rutschky sich seiner eigenen Zugehörigkeit zum literarischen Feld sehr sicher war, er konnte sich ihr lesend immer wieder aufs Neue versichern und brauchte sich nicht abzusichern, indem er sich über andere empörte.

Außerdem hatte er sich den Kulturpessimismus zum erklärten intellektuellen Feind erkoren. Er hegte geradezu einen Widerwillen gegen Verfallsdiganosen. Als die Talkshows im Fernsehen aufkamen, in den 90er-Jahren, wurden sie unter dem Signum Spaßgesellschaft analysiert, durchaus mit adornitischem Einschlag: Kulturindustrie!, lautete das Verdikt. Nun bewunderte Michael Rutschky vieles von Adorno, die Minima Moralia etwa, aber das Kulturindustrie-Kapitel aus der Dialektik der Aufklärung machte er nicht mit. Dafür ging er schon viel zu gerne ins Kino. Wenn Adorno länger gelebt hätte - vor allem auch die emanzipativen Errungenschaften ab den 70er-Jahren erlebt hätte -, hätte er es revidiert, hat mir Herr Rutschky einmal erzählt.

Von da aus eine Randbemerkung. Die Abwertung neuerer Zugänge zum Literarischen - etwa der berühmten Buchblogs - haben erstens, denke ich, ihren Zenit überschritten und zweitens nicht nur etwas mit dem Verlust privilegierter Zugänge, etwa der Literaturkritik, zu tun, sondern auch mit einem unsicheren eigenen Zugang zu den Büchern, der sich aus der vermeintlichen Entauratisierung des Fetischs Buch ergibt. Ein selbstbewusster Leser, der wirklich an dem Inhalt, der Geschichte interessiert ist, braucht andere nicht abzuwerten. Wohl aber jemand, der meint, sich mit dem Buch Distinktionen erkaufen zu können. Was im Übrigen keine Pauschalverteidigung von Literaturblogs ist; es kommt vielmehr stets auf den Einzelfall an, wie bei Literaturkritikern und Romanen ja auch.

\section{GLÜCK DES LESEKREISES}

Wie sicher sich Michael Rutschky seiner Zugehörigkeit zum Literarischen war, kann ich ganz gut bezeugen. Einige Jahre lang war ich ein Mitglied des Lesekreises um Michael Rutschky, selbstironisch auch Bibelkreis genannt, der sich alle vierzehn Tage, jeden zweiten Sonntag um 20 Uhr traf, um bei Weißwein, Wasser und Nüssen gemeinsam Bücher zu lesen. Im Wesentlichen haben wir uns philosophische Klassiker 
vorgenommen. Ich erinnere mich an inspirierende Lektüren von, ja auch Kant und Hegel, aber auch Tocqueville, Luhmann, Barthes, Aristoteles, Hume, William James, Martin Seel und manchen mehr - im Laufe der Jahre kam einiges zusammen.

So ein Lesekreis liefert viel Anschauungsmaterial, um zu erfahren, wie die anderen Menschen lesen, und dabei womöglich auch Typen von Lesenden zu charakterisieren. Was in dem Lesekreis zum Beispiel zu beobachten war, und zwar in allen möglichen Techniken und Intensitäten, waren Unterstreichungen und Kritzeleien an den Rand der Seite. Das war teilweise sehr lustig und ging bis zur Unterstreichung als Kunstform für sich.

Wie machte es Michael Rutschky? Sehr zurückhaltend. Er markierte in den jeweiligen Büchern Stellen, die er gern gelesen hatte, mit einem kleinen Kreuz am Rand, das war alles. Als ich das das erste Mal gesehen hatte, war ich ein Stück weit enttäuscht; das war zu einfach, es stand kein Geheimnis, keine erlernbare Technik hinter seiner Art, mit den Büchern umzugehen. Einen ähnlichen Effekt, nur noch etwas größer, gab es, als ich einmal im Untergeschoss des Hauses des berühmten Soziologen vor dem Zettelkasten von Niklas Luhmann stand. Man stellt sich als Student eine auratische Maschine vor, aber dann waren es - einfach nur beschriebene Zettel; wenn auch mit einem geheimnisvollen Verweissystem untereinander. Und bei Rutschky waren es eben nur Kreuzchen. Aber, so lernte ich allmählich, es steckte doch ein Erfahrungswissen dahinter.

Das Erfahrungswissen bestand darin, dass man Texte, wenn man gut über sie reden möchte, in das Gespräch hineinholen muss, will sagen: Man muss sie laut lesen. Und genau dazu dienten diese Kreuzchen. Sie markierten die Stellen, von denen Michael Rutschky annahm, dass sie sich lohnen würden, in der Gruppe laut vorgelesen zu werden. An die vorgetragenen Wörter konnte man dann mit eigenen Wörtern anknüpfen, mit Anmerkungen, Beispielen, Gegenthesen oder auch Sätzen wie „Das hat mich aber nicht überzeugt“ oder „Das habe ich nicht ganz verstanden“ oder „Dazu fällt mir ein, dass ...“

Mit einem George-Kreis hatte das alles nichts zu tun. Auch nichts mit den K-Gruppen-Kontexten, die im alten Westberlin viel naheliegender als Vergleich wären. 
Es gab selbstverständlich gruppendynamische Prozesse, manchmal wurde auch laut gestritten. Aber der Kreis war offen, wer jemanden mitbringen wollte, konnte das tun.

Seitdem ich in diesem Lesekreis war, kann ich ein Stück weit nachvollziehen, warum es im deutschsprachigen Literaturbetrieb so ein erstaunliches Bedürfnis nach Präsenz gibt. Das laute Lesen macht etwas. Lesend machte man den Text zu einem Bestandteil des Gesprächs, man aktualisierte ihn auch ein Stück weit. Man wollte schon herauskriegen, was in dem jeweiligen Buch steht, es ging schon um hermeneutische Arbeit, aber man wollte das Buch oder den Autor sozusagen nicht aufspießen oder abheften. Man wollte sich untereinander keine originelle oder fertige Interpretation des Textes bieten, sondern sich miteinander mit dem Text ins Verhältnis setzen.

Auf Literaturfestivals und Leseevents stört mich dieses Bedürfnis nach Präsenz oft, ich wittere dann schnell ein voyeuristisches Interesse an der Person der Autor*in. Aber im Fall des Lesekreises ging es eben um eine Präsenz des Textes, ich glaube, wenn die Autor*in real anwesend gewesen wäre, hätte uns das eher gestört, weil es das Gespräch zu sehr auf eine Perspektive fokussiert hätte. Die schönsten Lesestunden waren die, wenn unterschiedliche Teilnehmer*innen des Kreises verschiedene Aspekte des jeweiligen Textes herausstellen und dann auch nebeneinander stehen lassen konnten. Es ging ja darum, in der Auseinandersetzung mit dem Text immer auch die eigenen Grundannahmen und Hintergründe bestätigt oder auch hinterfragt zu sehen. Und es ging darum, von den anderen Teilnehmer_innen des Lesekreises zu erfahren, was man auch noch über den Text denken kann, über den jeweils eigenen Horizont hinaus. Ohne das jetzt romantisch verbrämen zu wollen, es hatte schon etwas Ideales, wenn sich der Reichtum eines Textes in einer Vielzahl von Perspektiven auf ihn realisierte. Das war nichts, was einem das einsame Lesen zu Hause auf dem Sofa ersetzen konnte, im Gegenteil, man musste sich durchaus selbst eine eigene Perspektive auf den Text erarbeitet haben. Aber es war etwas, was einen dazu anspornen konnte, das auch tatsächlich zu tun, und was einen zugleich mit einem ganzen Arsenal möglicher Reaktionen anderer auf die eigene Perspektive ausstattete.

Das alles war kein ausformuliertes Programm, man redete auch kaum auf einer Metaebene, warum man wie in dem Lesekreis liest. Es war ein über Jahre 
eingespieltes Verfahren, und es funktionierte gut. Mehr noch, es war teilweise sogar beglückend.

Das für mich Interessante ist nun, dass ich eigentlich gar keine richtige Sprache vorfinde, um das Positive an diesen Lektüreerfahrungen kitsch- und stolperfrei aufzuschreiben. Man kennt natürlich diese abschreckenden Marketingkampagnen, mit denen in Deutschland für das Lesen geworben wird. Wellness für die Seele und solche Sachen. So möchte man es natürlich nicht machen.

Immerhin gibt es auch Ansätze, das besser zu beschreiben. In der Studie Die Unruhe der Bücher von Sascha Michel steht der so pathetische wie vielleicht schlicht auch zutreffende Satz: „Genau dafür brauchen wir die Bücher: damit uns immer wieder schockartig bewusst ist, wie viel größer das Universum ist, als wir es zu denken gewohnt sind. “" ${ }^{3}$ Lesen ist für Michel ein Herd der Unruhe und Kontingenz und eben gerade nicht eine kontemplative Quelle der Ruhe und Entschleunigung.

Im New Yorker stieß ich auf einen Essay des Journalisten Joshua Rothman, der sich auf die Kulturgeschichte „Loving Literature“ der Literaturprofessorin Deidre Shauna Lynch bezieht. ${ }^{4}$ Entscheidend ist für sie, dass Lesen „die Distanz zwischen einem selbst und den anderen sowie zwischen dem Jetzt und dem Dann“ überbrückt. Lesen, so verstanden, heißt also nicht Seelenmassage oder Wellness jenseits der Zerrissenheit der Welt, sondern vielmehr Kommunikation, Kontaktaufnahme und ein Gefühl dafür, dass die Welt größer ist als das Zimmer, in dem man liest.

Auch Kanon-Ideen kann man mit Rothman und Lynch anders werten als in Deutschland üblich. Ein literarischer Kanon kommt bei ihnen vor, bildet aber keinen festen Felsen, um darauf Identitäten zu bauen. Stattdessen wäre es besser, von variablen Gravitationspunkten auszugehen, um die Praxis literarischer Kulte zu ermöglichen: Kulte um Jane Austen, James Joyce, Leo Tolstoi, Franz Kafka, Thomas Bernhard, Ingeborg Bachmann, neuerdings womöglich Karl Ove

${ }^{3}$ Sascha Michel: Die Unruhe der Bücher. Vom Lesen und was es mit uns macht. Stuttgart 2020, S. 47

${ }^{4}$ Joshua Rothman: The history of "loving" to read. The New Yorker, Ausgabe vom 2. 2. 2015 
Knausgård. Das trägt den ständig stattfindenden Verschiebungen im literarischen Feld Rechnung.

Vielleicht kann der Börsenverein des Deutschen Buchhandels von seiner Wellnessidee wirklich bald einmal herunterkommen; aber ich bezweifle es. Der deutsche Buchhandel ist unglaublich konservativ. Ich glaube, die Vorstellung, dass Lesen auch beunruhigen könnte, löst da Panik aus.

Eins der Bücher, die wir im Lesekreis von Michael Rutschky mit großer Zustimmung gelesen haben, war die Essaysammlung Tiefer hängen des Kunsthistorikers Wolfgang Ullrich. Wolfgang Ullrich schlägt vor, und man kann sich das ganz konkret vorstellen, Kunstwerke tiefer zu hängen, um mit ihnen auf die gleiche Augenhöhe zu kommen. Der Gewinn dieser Operation besteht darin, dass man dann, wenn man nur zu lesen versteht, Erfahrungen mit dem Kunstwerken abgleichen kann. Und das haben wir in dem Lesekreis dann auch gemacht.

Insofern ist es vielleicht nicht zufällig, dass ich im Umfeld von Michael Rutschky zum ersten Mal systematisch von einem Genre hörte, das in den vergangenen zehn Jahren das literarische Feld ziemlich aufgemischt hat: Ich meine den Trend zur Autofiktion, also von seinen eigenen Erfahrungen aus zu erzählen. Karl Ove Knausgard, Annie Ernaux, Didier Eribon oder im Deutschen etwa Christian Baron. Die Art Texte scheint gegenwärtig auf einen Nerv bei den Leserinnen und Lesern zu treffen.

Im Umfeld von Michael Rutschky konnte man gut vorbereitet auf diese Entwicklung sein. Bei Michael Rutschky hießen Bücher, die sich zwischen Sachbuch und Literatur, zwischen Roman und Autobiografie nicht eindeutig zuordnen lassen, „Bücher ohne Familiennamen“. Und seine eigene essayistische Technik lässt sich in die fast ein bisschen geheime Vorgängergeschichte der Autofiktion einordnen. In seinen Essays ging er gern von konkreten Erfahrungen aus, um sie hermeneutisch durchzuarbeiten und abzuklopfen.

Der Punkt ist vielleicht auch, dass die Unterscheidung zwischen fiktiv/nicht fiktiv für ein Lesen auf Augenhöhe möglicherweise gar nicht so wichtig ist. 
Mit Michael Rutschky - und jetzt meine ich den Autor Michael Rutschky, nicht den Leser - lässt sich das auch theoretisch beglaubigen. In seinem einschlägigen Essayband Lebensromane aus dem Jahr aus dem Jahr 1998 (einem der vielleicht unterschätztesten Essaybände der jüngeren Vergangenheit) hat er anschaulich gemacht, dass menschliche Lebensläufe oft literarischen Mustern und Dramaturgien folgen, dass das Fiktive also gewissermaßen in die Realität eingebaut ist.

Im Hintergrund arbeitete auch John Deweys Studie Kunst als Erfahrung, in der es um den Gedanken geht, dass eine Erfahrung, erst dann tatsächlich gemacht ist, wenn man sie auch erzählen kann. Auch das treibt möglicherweise den gegenwärtigen Boom von Autofiktionen an. Es ist eben keine Anmaßung, von sich zu erzählen, oder keine Selbstbespiegelung, oder wie die Vorwürfe auch immer heißen. Im Kern geht es eher darum, die Erfahrungen, die man in dieser Gesellschaft gemacht hat, erzählbar werden zu lassen - und so erst wirklich zu machen: Auch die Erfahrung des Lesens ist erst gemacht, wenn man von ihr erzählen kann.

Ich frage mich, ob man über dieses Abklopfen von Büchern auf die Erfahrungen, die in ihnen formuliert werden, herauskommen kann aus den Distinktionsspielen rund um Bücher. Eine Antwort auf diese Frage habe ich noch nicht.

\section{DIE NACHTSEITE DES LESENS}

Das, was ich bislang hier geschrieben habe, betrifft die Tagseite des Lesers Michael Rutschky und was für mich von ihr anschlussfähig ist. Es gibt aber auch eine Nachtseite, und erst wenn man sie auch in den Blick nimmt, ergibt sich das ganze Bild von dem existenziellen Ernst, den das Lesen für ihn hatte - und den man, wenn auch vielleicht nicht in diesem Ausmaß wie bei Herrn Rutschky, Leseschaften zumindest als prinzipiell möglich unterstellen kann.

Das mit der Nachtseite meine ich ganz wörtlich. Wie Herr Rutschky in privaten Gesprächen gelegentlich andeutete und wie man es auch in seinen veröffentlichten 
Tagebüchern nachlesen kann - vor allem im dritten, postum veröffentlichten Band ${ }^{5}$-, konnte Herr Rutschky nachts oft nicht schlafen. Angstgefühle. Depressive Anflüge. Solche Sachen.

Was hat er getan? Unter anderem zur Beruhigung Romane gelesen. Ich muss hier etwas spekulieren, weil mir das Material fehlt (Herr Rutschky war in privaten Mitteilungen etwas spröde, man bekam sie nur gefiltert), aber ich meine, dass in diesen einsamen und teilweise bedrängten nächtlichen Lesestunden noch ein anderer Leser auftritt. Über Marcel Prousts Suche nach der verlorenen Zeit - er hat die Bände bestimmt vier- oder fünfmal gelesen - hat Michael Rutschky zum Beispiel eine Bemerkung gemacht, die mich aufhorchen ließ. Die ganze ausufernde Heldenreise der Suche nach der verlorenen Zeit sei, so Herr Rutschky, nichts anderes als der Versuch des Erzählers, das Verlassenwerden durch die Mutter, nachdem sie ihm gleich zu Beginn den berühmten Gutenachtkuss gegeben hat, herauszuschieben.

Eine viertausendseitige hochkomplexe Romanwelt voller Gesellschaftsbeschreibungen, Porträts und feinst ausgemalter Bewusstseinsaufnahmen nur um nicht allein einschlafen zu müssen. Wie kommt man auf so einen Gedanken? Letztlich glaube ich von ihm ausgehend, dass selbst so ein rationales, selbstreflektiertes und versiertes Lesen wie das von Michael Rutschky ein Stück auf einem fast metaphysischen Punkt ruht.

Ich möchte mich, um diesen Punkt zu beschreiben, ein bisschen hinter dem Autor Wolfgang Herrndorf verstecken. Wolfgang Herrndorf hat dieses Gefühl, das Herr Rutschky in seinen nächtlichen Stunden suchte, in seinem Buch Arbeit und Struktur so beschrieben: „[...] dass man teilhat an einem Dasein und an Menschen und am Bewusstsein von Menschen, an etwas, worüber man sonst im Leben etwas zu erfahren nicht viel Gelegenheit, selbst, um ehrlich zu sein, in Gesprächen mit Freunden nur selten“.

${ }^{5}$ Michael Rutschky: Gegen Ende. Tagebuchaufzeichnungen 1996-2009. Berlin 2019 
Wieder also: Teilhabe, Zugehörigkeit, nur anders als bei Deniz Ohde, nicht konkret gesellschaftlich, sondern eher die letzten Dinge berührend. Anders gesagt, auch Michael Rutschky und Wolfgang Herrndorf, diese kritischen, oft auch spöttischen Geister, wollten sich durch Lesen existenziell trösten lassen.

Ansätze, den Trost der Literatur zu verkitschen oder marktmäßig auszubeuten, gibt es genug, einige habe ich erwähnt. Ich wäre sehr denkbar für andere Ansätze oder Sprechweisen, nämlich für solche, die es ermöglichen, ihn beim Nachdenken über Lesen und Schreiben stets im Hinterkopf behalten zu können.

Vielleicht ist genau dies das Heraustreten aus dem Distinktionsspiel. Aber vielleicht ist das auch nur eine Literaturkritikerfloskel. 


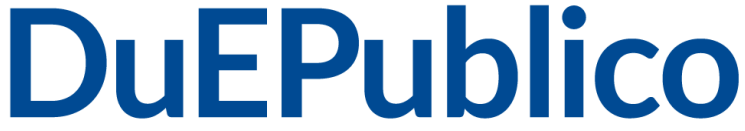

Duisburg-Essen Publications online
UNIVERSITÄT

DE ${ }^{\prime} S_{S} S^{B} E N$ U R G

offen im Denken

$\mathbf{u b} \mid \begin{gathered}\text { universitäts } \\ \text { bibliothek }\end{gathered}$

Dieser Text wird via DuEPublico, dem Dokumenten- und Publikationsserver der Universität Duisburg-Essen, zur Verfügung gestellt. Die hier veröffentlichte Version der E-Publikation kann von einer eventuell ebenfalls veröffentlichten Verlagsversion abweichen.

DOI: $\quad 10.37189 /$ duepublico/74191

URN: urn:nbn:de:hbz:464-20210716-102758-4 